\title{
Textures Recognition using Elman Neural Network
}

\section{Fawziya Mahmood Ramo \\ Alaa Anwr Mohamed}

Fawziya.ramoo@uomosul.edu.iq

College of computer science and mathematics

University of Mosul, Iraq

\section{Received on : $26 / 2 / 2013$}

Accepted on : 24/6/2013

\begin{abstract}
In this research building system to recognition texture images using artificial neural networks. The system consists of two phases: phase extraction important feature of each texture by using an algorithm Principal Components Analysis (PCA) and recognition phase which recognize these feature by using Elman network were trained network on a number of various texture models down to the steady-state network and then test the network by input samples of textures. The experiments show that the method achieves high performance and produces $92 \%$ recognition rate.

Key words : pattern recognition, Neural network, feature extraction, Texture images

$$
\text { فوزية محمول رموا }
$$

$$
201316124 \text { : تاريخ قبول البحث }
$$

201312126 : تاريخ استلام البحث

\section{الملخص}

تم في هذا البحث بناء نظام لتمييز صور الانسجة باستخدام الثبكات العصبية الاصطناعية. يتكون النظام من مرحلتين مرحلة استخلاص الخصائص المهمة من كل نسيج عن طريق استخدام خوارزمية تحليل المركبات

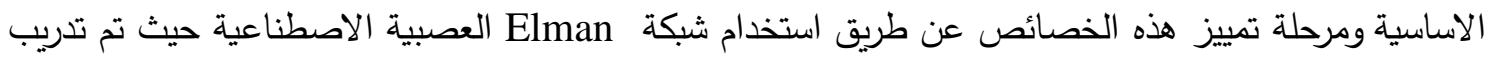
الثبكة على عدد من نماذج الانسجة المتتوعة وصولا الى حالة استقرار الثبكة ومن ثم اختبار الثبكة بإدخال

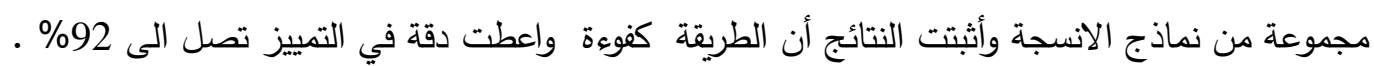
الكلمات المفتاحية : تمييز الانماط ، شبكات العصبية الاصطناعية ، استخلاص الخصائص، صور انسجة
\end{abstract}

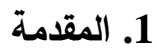

تطبيقات معالجة الصور تستخدم إما لتحسين الصور وتمهيدها ودعمها لعمليات معالجة لاحقة أو لتحليل

الصور واستخلاص بعض المعلومات منها ليتمكن الحاسوب من إدراكها وتقسيرها. وقد ظهرت الحاجة إلى تحليل

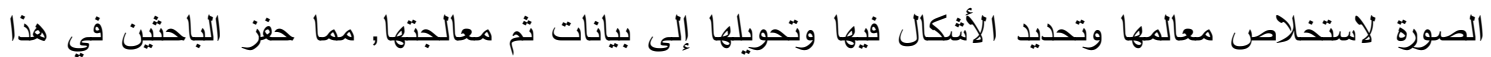
المجال إلى تقديم عدد من الخوارزميات المقترحة لتحديد الأشكال وتمييز الخصائص في الصورة والتي تعتمد على ولى التطبيق المستخدم, ويرتبط تحليل الصور مع استخلاص مقاييس مفيدة أو بيانات أو معلومات عن الصورة عن طريق أجهزة وأنظمة آلية وشبه آلية والتي تثتمل على معالجة بيانات الصورة لتحديد المعلومات المطلوبة الضرورية لحل مشكلة الإبصار الحاسوبي[11]. إن مجال التمييز من المجالات الواسعة والمهمة والتي كانت محط اهتمام العديد من الباحثين في أدناه عدد

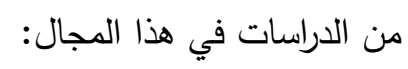


في عام 2005 قام كل من Imran Sarwar Bajwa و S. Irfan Hyder من معهد كراتثي للاقتصاد و التكنولوجيا, بيناء نظام يستطيع تمييز صور الغيوم. يعتمد هذا النظام على تحليل المركبات الأساسية لاستخلاص

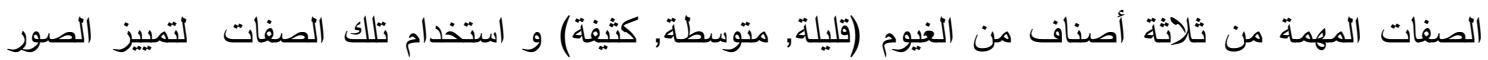
الداخلة إلى النظام[2]. في عام 2010 قدم (Y.H. Zhang) نظام ذكي لاكتثاف خلل نسيج وتمييز نوع الخلل بالاعتماد على كابور فلتر (Gabor Filter) و شبكة ايلمان العصبية(Elman Neural Network) [3]. في

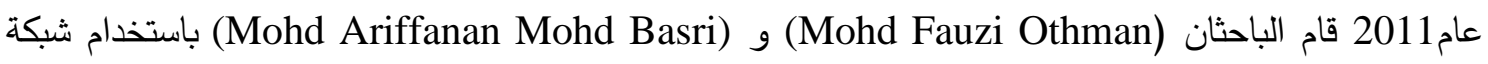
Principal ) وخوارزمية تحليل المركبات الاساسية (Probabilistic Neural Network PNN)

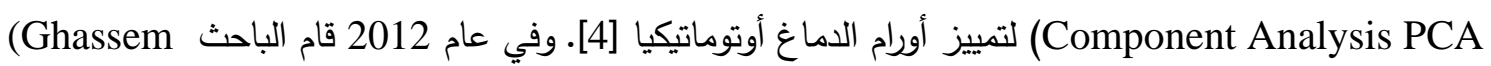
بتطبيق نظام لكثف وصول أجرة البريد حيث تم استخدام خوارزمية تحليل المركبات الاساسية وجعلها إدخال للشبكة العصبية الاصطناعية لعمل توقعات لمرور أجرة البريد[5].

\section{2. هدف البحث}

يهدف البحث إلى تصميم نظام له القدرة على تمييز الأنسجة باستخدام تقنيه ذكاء اصطناعية معتدة على الثبكات العصبية الاصطناعية بعد استخلاص خصائص النسيج باستخدام خوارزمية تحليل المركبات الاساسية التي تعد احدى طرائق استخلاص الصفات او الخصائص واعتماد البحث نواة لأنظمه مستقبليه تعتمد على أستخدام الأنسجة للتصنيف أو تمييز أوتقطيع الصور في المجالات الطبية والعلمية الأخرى.

3. استخلاص الخصائص(Features Extraction)

استخلاص الخصائص تعرف كأداة رياضية للفصل بين البيانات وهي عادة تعتبر مكمل في معظم أعمال تمييز الأنماط. تشمل اشتقاق مجموعة من الخصائص أو المتجهات التي تتضمن اختلافات هامة تتعلق بتركيب البيانات حيث تخفض أبعاد وحجم مثل هذه البيانات[6]. إن استخلاص الخصائص من المراحل المهمة في نظام التمييز حيث تأخذ الصفات التي تحقق دقة عالية في التمييز وسرعة في المعالجة[7].

4. تحليل المركبات الأساسية (Principal Components Analysis PCA)

تحليل المركبات الرئيسية يسمى كذلك تحويل المركبات الرئيسية ،تحويل متجه (Eigen). قدم هذا التحويل لأول مرة من قبل بيرسن ( 1901 ) وتمت عليه تحويرات عدة إلى أن تم تعميمه من قبل لوي (1963) [8]. تعتبر طريقه لتمييز الأنماط في البيانات. ويُظهر البيانات بطريقه حيث يميز الثيء المهر بالنسبة للتشابه والاختلاف في هذه البيانات, حيث يمكن لخوارزمية تحليل المركبات الاساسية ان تحول سلسلة البيانات المترابطة بعض الثيء الى سلسلة مرتبة من المكونات الاساسية توفر وسيلة لاستخلاص الخصائص. أن انجاز التحويل

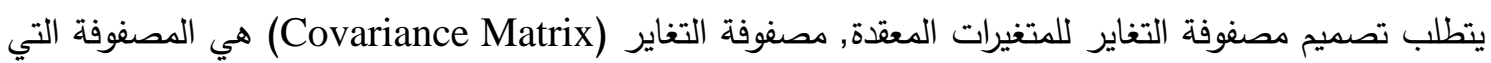
يكون عناصر قطرها الرئيسي هي الخلاف (Variance) للمتغيرات, وعناصر القطر الثانوي (Off-Diagonal)

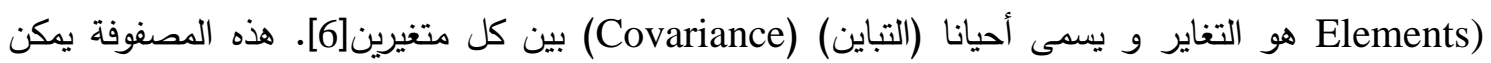

$C_{i j}=\frac{1}{M N} \sum_{k=1}^{M} \sum_{l=0}^{N}\left(X_{i}(K, l)-\mu_{i}\right)\left(X_{j}(K, l)-\mu_{j}\right)$ حسابها بالصيغة التالية : 


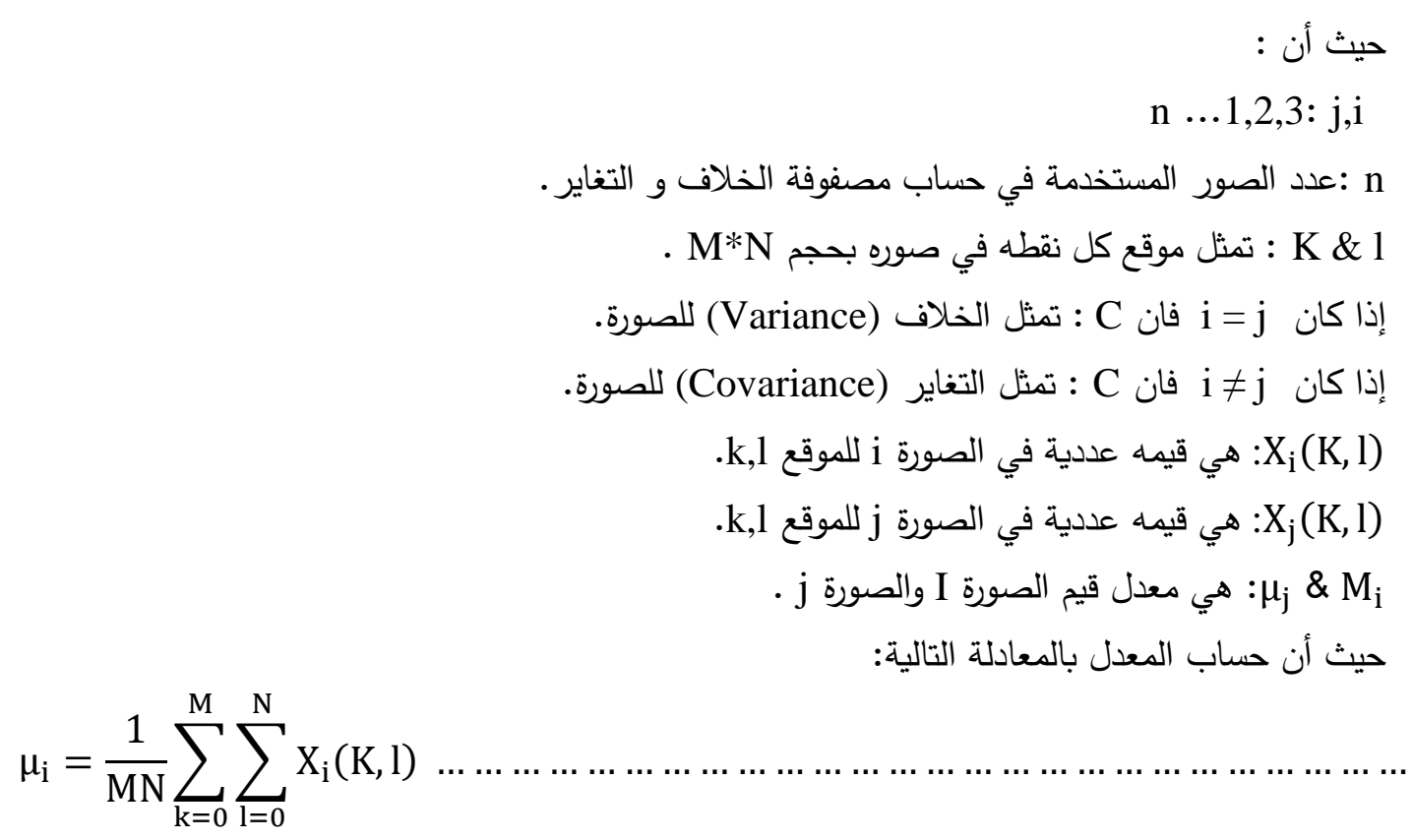

مصفوفة آيكن (Eigen-Vector \& Eigen-Value matrix):

هي مصفوفة مشتقة من مصفوفة التغاير (Covariance Matrix) باستخدام نظرية جاكوبي لإيجاد

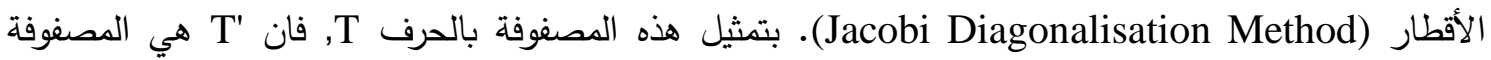
المدورة[8]. كما في المعادلة(3) التالية:

$\mathrm{D}=\mathrm{TCT}^{\prime}$

حيث أن المصفوفة D تمثل قطر مصفوفة التغاير (Covariance Matrix) ويمكن تمثيلها بالمصفوفة

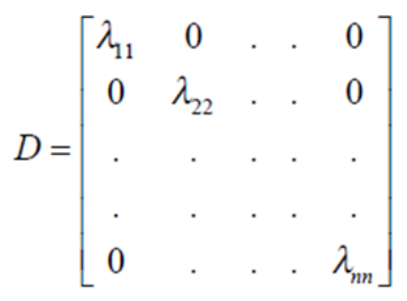

العناصر القطرية ( Diagonal Elements) للمصفوفة D تدعى قيم آيكن (Eigen-Values) لمصفوفة

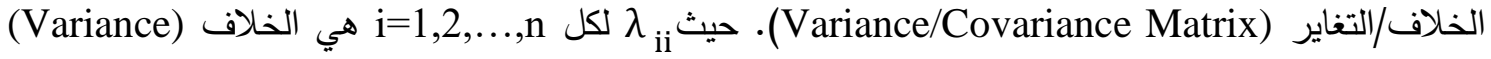
لا(PCA).وباقي العناصر (Off-Diagonal) للمصفوفة D تحمل القيمة (Zero 0) مما يدل على أن المركبات الخارجة غير مترابطة, أي أنها مستقلة [8]. بعد حساب قيم المصفوفة T, يمكن إجراء التحويل للصورة باستخدام ألصيغه التالية: $\mathrm{Y}=\mathrm{TX}$ 
[8] (Eigen-Vector Matrix) قيم مصفوفة T

5. الثبكة العصبية الاصطناعية(Artificial Neural Network)

الثبكات العصبية هي تقنيات حسابية مصممة لمحاكاة الطريقة التي يؤدي بها الدماغ البشري مهمة معينة، ومكونة من وحدات معالجة بسيطة،هذه الوحدات ما هي إلا عناصر حسابية تسىى عصبونات أوعقد والتي لها خاصية عصبية، من حيث أنها تقوم بتخزين المعرفة العملية والمعلومات التجريبية لتجعلها متاحة للمستخدم وذلك عن طريق ضبط لهات الأوزان. ويتم ربط هذه العصبونات بواسطة بعض أنواع الربط والتي تحمل بيانات رقمية. تستخدم الثبكات العصبية الاصطناعية في مجالات علمية مختلفة منها التمييز الصوري، وإيجاد قيم الدالة التقريبية، والتخطيط لإيجاد حل

امثل للمسائل، مجالات التشخيص الطبي والسيطرة والتحكم [9].

1.5

تعد شبكة ايلمان العصبية من الثبكات العصبية التي طورت من قبل جيفري ايلمان عام 1990 وتعد من شبكات التدريب باشراف وتمتلك اعادة تغذية (recurrent) [10]. هذا النوع من الشبكات يتكون على الاقل من من فئل ثلاث طبقات طبقة الادخال والطبقة المخفية وطبقت الاخراج بالاضافة الى طبقة context layer التي تعمل تغذية راجعة بدون وزن، ان هذه الثبكة تمتاز بقدرة تمييز عالية ووصولها إلى الحل الأمثل لأنها تعيد اخراج الطبقة

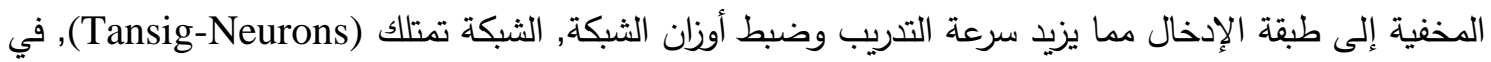
الطبقة المخفية وكذلك تمتلك (Purelin-Neurons) في طبقة الإخراج. كما في الثكل (1)الذي يوضح معمارية

الثبكة.[11]

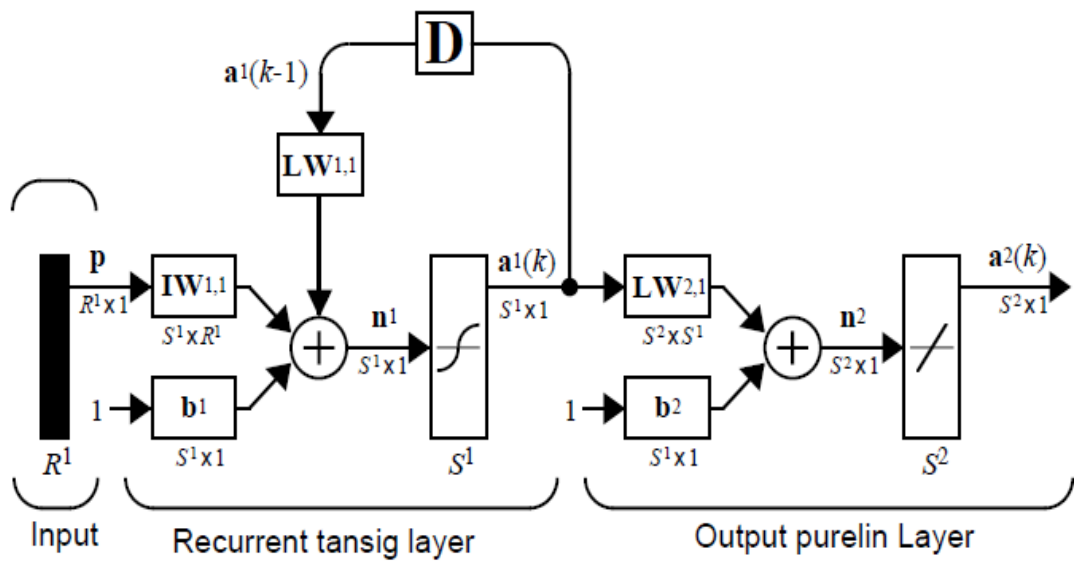

ELMAN الثكل (1): معمارية شبكة

S:

S:

IIW: مصفوفة الوزن للطبقة المخفية.

L LW2,1 مصفوفة الوزن لطبقة الإخراج.

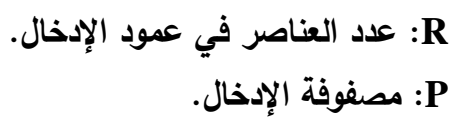

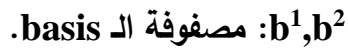

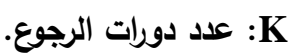

العقد في الطبقة المخفية تتفذ بواسطة الدالة (tansig) الرجعية التي تعمل على منطقة محصورة من حيز

الإدخال. وذلك باعتماد المعادلة الآتية[11]: 


$$
a 1(k)=\tan \operatorname{sig}\left(I W_{1,1} p+L W_{1,1} a_{1}(k-1)+b_{1}\right)
$$

والعقد في طبقة الإخراج تنفذ بواسطة الدالة(purelin) الخطية التي تعمل على منطقة محصورة من حيز الإخراج. وذلك باعتماد المعادلة الآتية[11]:-

$$
a 2(k)=\operatorname{purelin}\left(L W_{2,1} a_{1}(k)+b_{2}\right)
$$

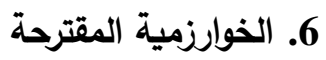

تم تصميم نظام تمييز انسجة الصور الرقمية المعتمد على تحليل المركبات الأساسية لاختيار أفضل الميزات من البيانات واستخدام شبكة (ELMAN) العصبية الاصطناعية لتمييز هذه الصور، النظام يعمل بثلاث مراحل مرحلة المعالجة الاولية، مرحلة استخلاص الخصائص ومرحلة التمييز كما موضح في المخطط الصندوقي الآتي :

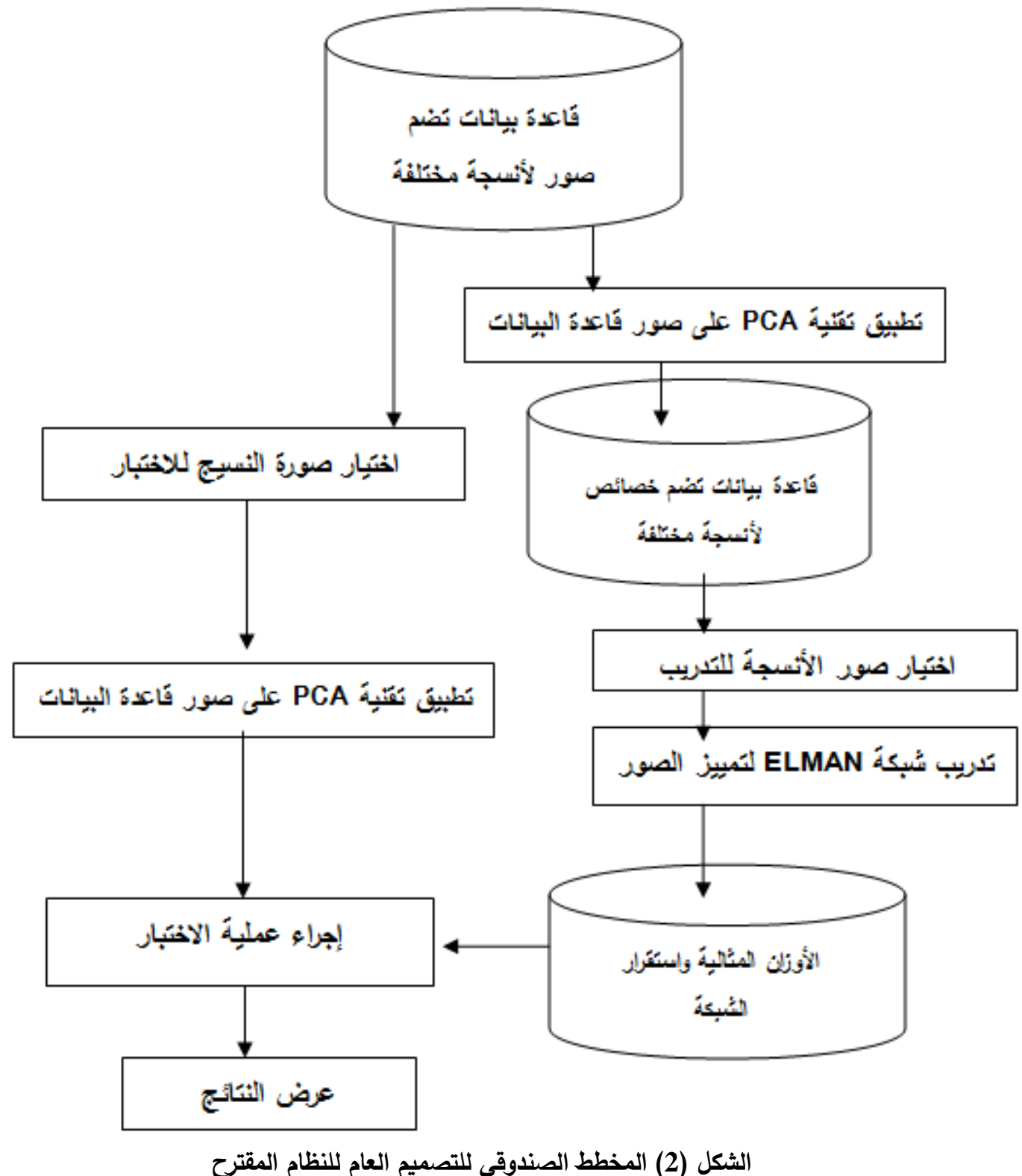

الثكل (2) المخطط الصندوقي للتصميم العام للنظام المقترح 
1.6. مرحلة المعالجة الاولية Preprocessing stage تتكون هذه المرحلة من الخطوات التالية : 1- تكوين قاعدة بيانات تضم نماذج لصور أنسجة مختلفة تمثل 10 أصناف متتوعة مثل ( نسيج الخشب المسطح، نسيج الحجر، نسيج الفاكهة،، نسيج الطابوق نسيج نجارة الخشب، نسيج أرضية منزل، نسيج ورق لهن

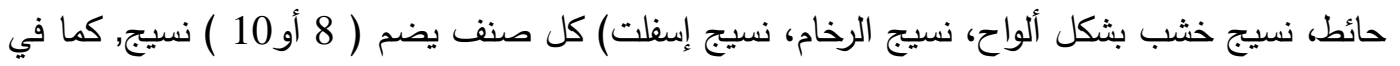
الثكل التالي: nen

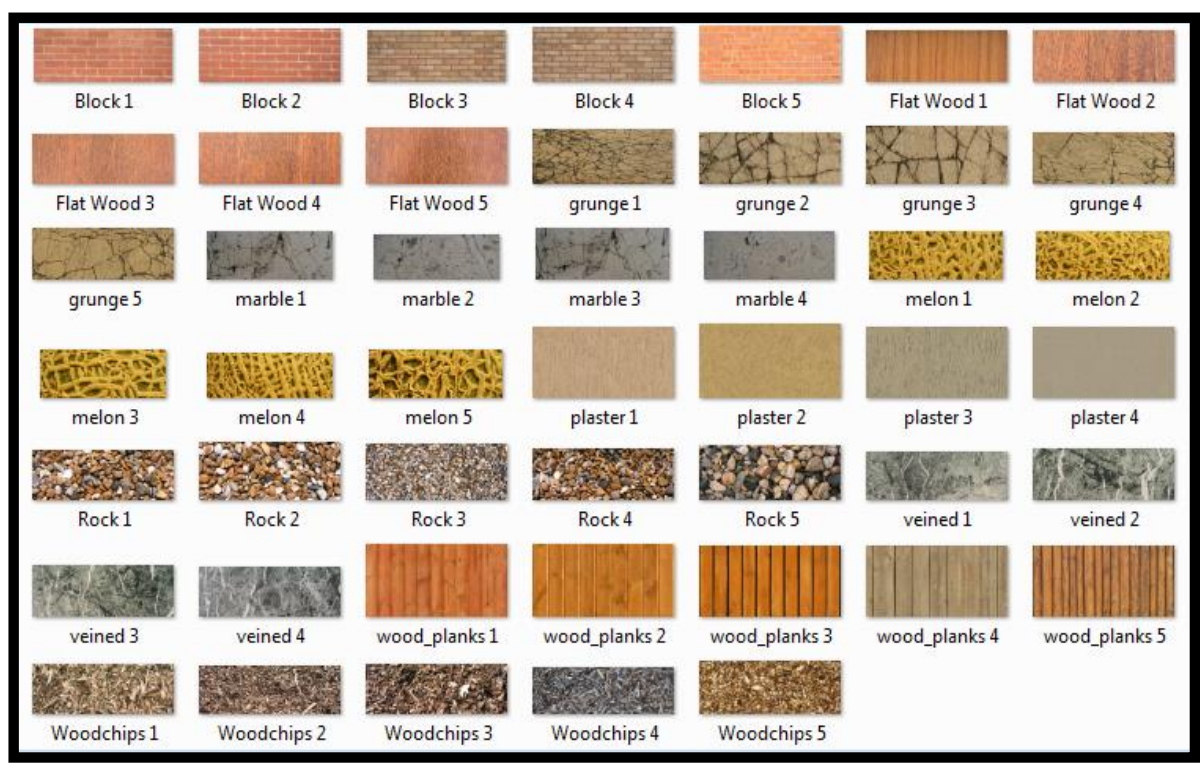

الثكل(3): يوضح نماذج ألانسجة لإنثاء قاعدة البيانات

2- تغيير حجم صور الأنسجة الى (512*312) كما في الثكل (4).

3- تحويل الصورة إلى فضاء الرمادي (Gray Level) كما في الثكل (5).

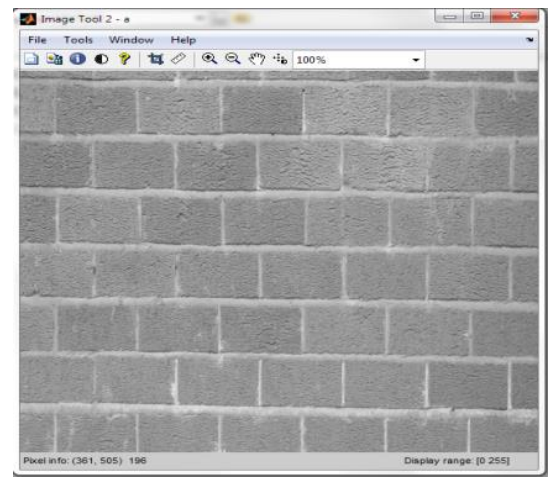

الثكل(5): النسيج في الفضاء الرمادي

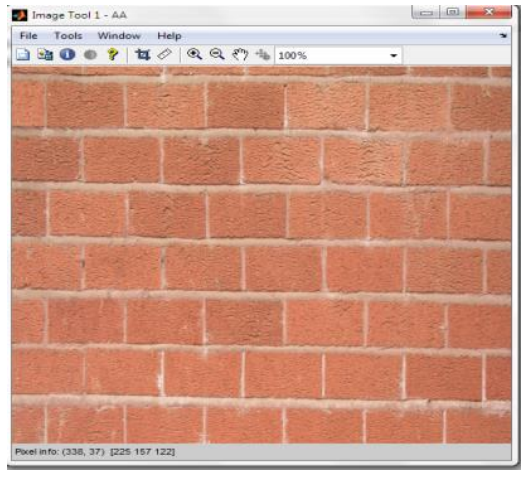

الثكل (4): نسيج بحجم (512*512)

2.6. مرحلة استخلاص الخصائص: تتفيذ خوارزمية تحليل المركبات الاساسية التي تتكون من الخطوات

$$
\text { * حساب المتوسط لقيم صور النسيج. }
$$

* حساب مصفوفة التغاير/الخلاف (Variance/Covariance Matrix).

* إيجاد مصفوفة أيكن (Eigenvalues And Eigenvectors Matrix). 


$$
\text { " إيجاد المعاملات (Coefficients) للبيانات التي تم اختيارها. }
$$

يتم خزن معاملات تحليل المركبات الاساسية لكل الانسجة في مصفوفة وخزنها في ورقة أكسل كما يوضح الثكل التالي :- (Excel)

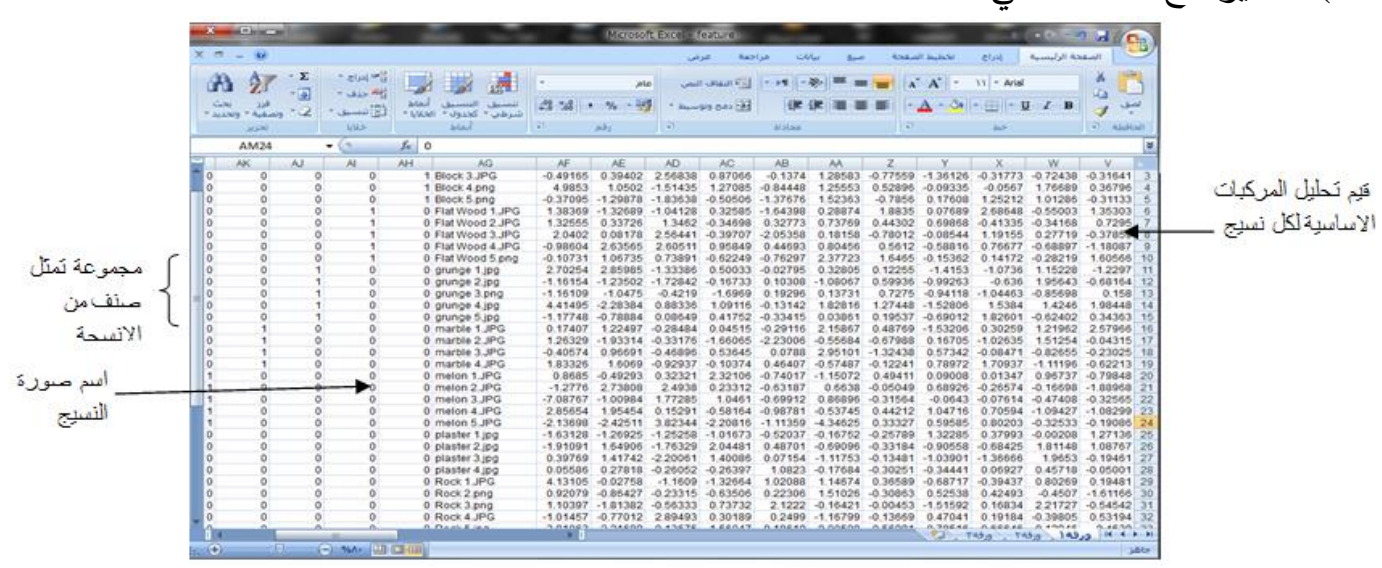

الشكل(6): جزء من قاعدة البيانات

3.6. مرحلة تمييز الانسجة: تتكون هذه المرحلة من طورين

طور التدريب حيث إن مرحلة تدريب الثبكة العصبية هي من أساس النظام, حيث تم هنبيز الناء شبكة (ELMAN)

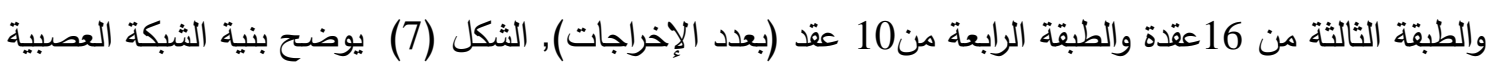
الاصطناعية من عدد الإدخالات, وعدد الطبقات, وعدد اخراجات الثبكة العصبية الاصطناعية حيث تم التدريب الثبكة على عدد من النماذج بلغت (47) نموذج وبعدد من الطبقات المختلفة وعدد من العقد المختلفة لجميع الطبقات وتم تدريب الثبكة الى ان يتم الوصول الى حالة الاستقرار والوصول الى افضل النتائج للشبكة. Layer

\section{الشكل (7): بنية الشبكة العصبية}

كان عدد دورات التدريب 1000, وكان متوسط تربيع الخطأ (0.00001). ولكل تدريب وقت مختلف مع نفس الثبكة العصبية ونفس البيانات, استغرقت الثبكة العصبية أثناء التدريب دقيقة وأربعة وخمسين

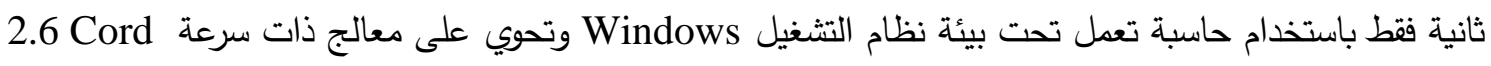

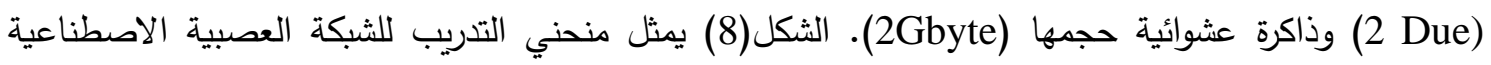




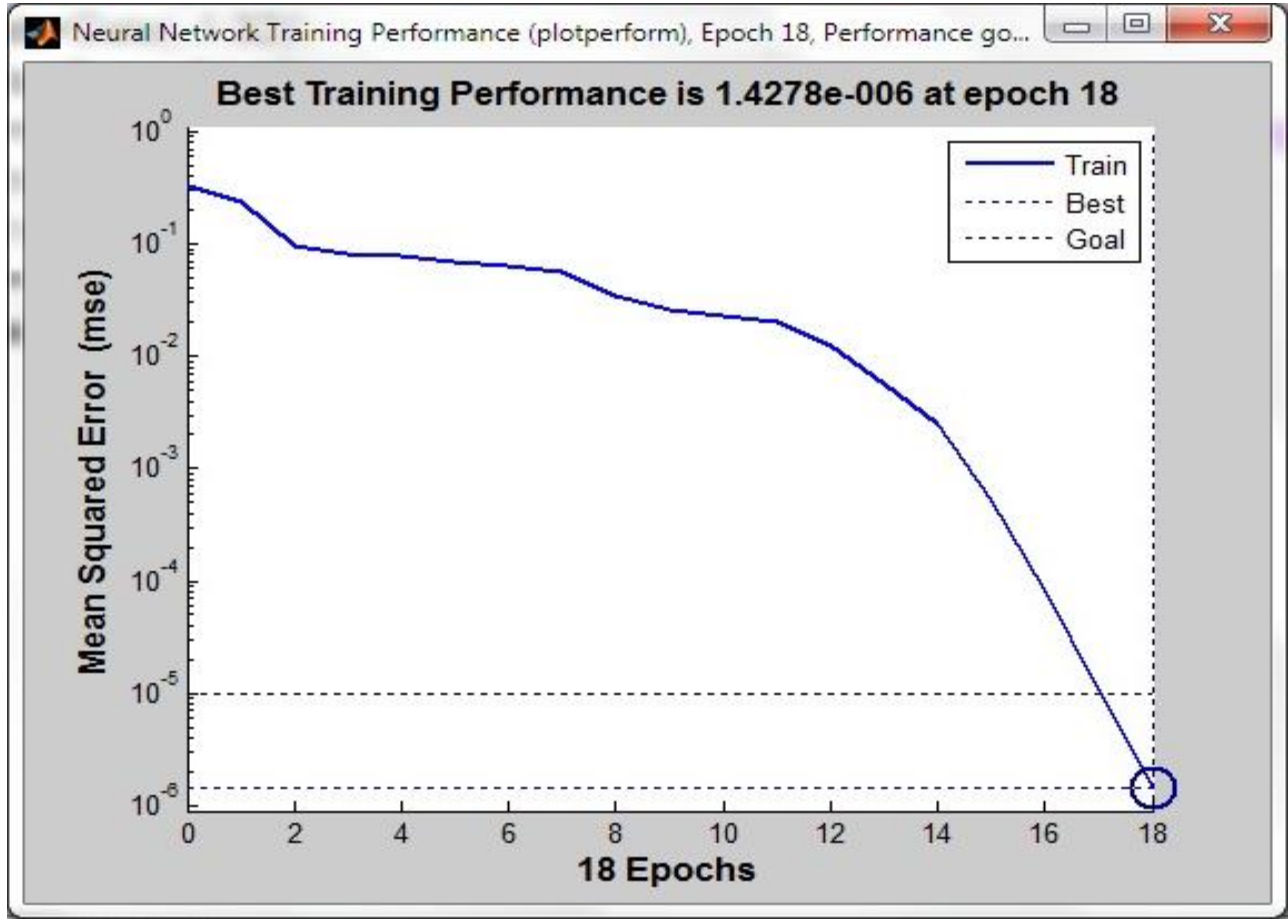

الثكل(8): منحني تدربب الشبكة

طور الاختبار حيث يبدأ بتجميع بيانـات الاختبار المتكونـة (47) نموذج وتهيئتها وإدخالها إلى الثبكة العصبية المدربـة مسبقا (الشكل(9) يمثل نمـاذج الاختبار) بعد الحصول على النتائج يتم تقييم أداء الثبكة عن طريق حساب نسبة التمييز حسب المعادلة التالية [11]:-

Recognition Rate $(R R)=T P / P$

TP تمثل عدد الصور المصنفة بشكل صحيح. P

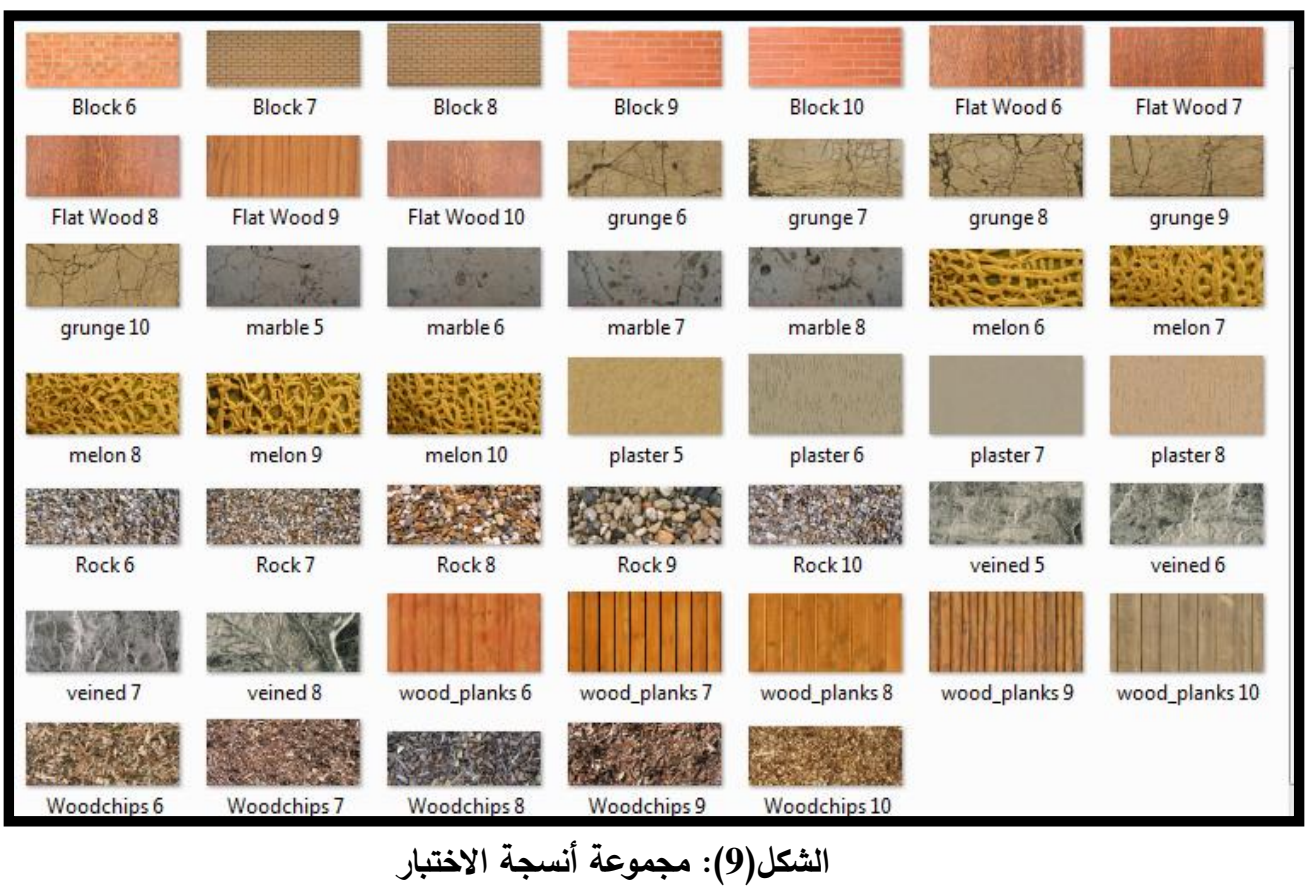


7. تنفيذ النظام ومناقشة النتائج

تم بناء عدة واجهات لتنفيذ النظام حيث يتم اختيار النسيج كما في الثكل (10) :-

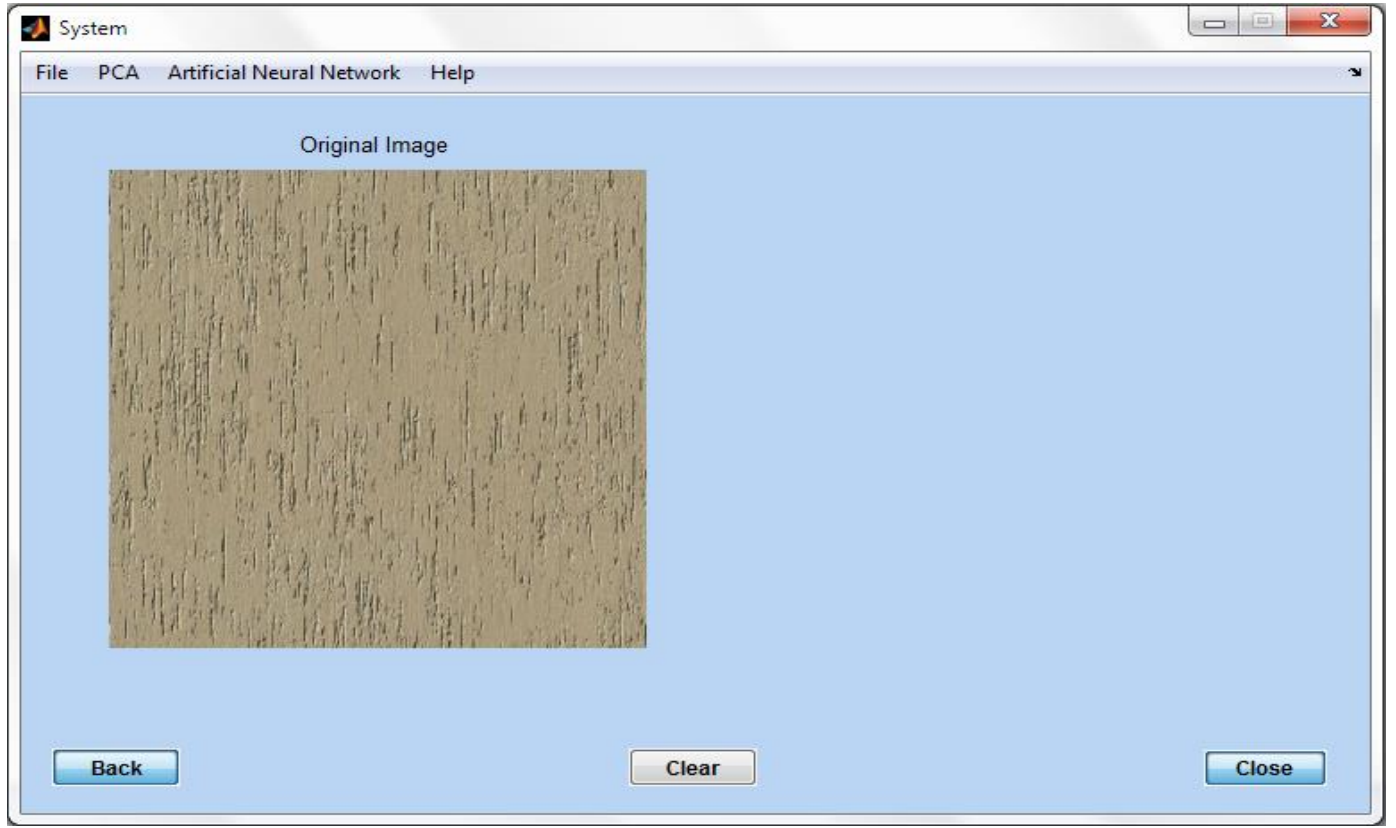

الثكل (10):الواجهة بعد استقبال الصورة

في الثكل (11) نتيجة تتفيذ مرحلة استخلاص الخصائص ورسم مخطط بياني يوضح معاملات تحليل المكونات الأساسية (PCA_Coefficients) للصورة التي تم اختيارها.

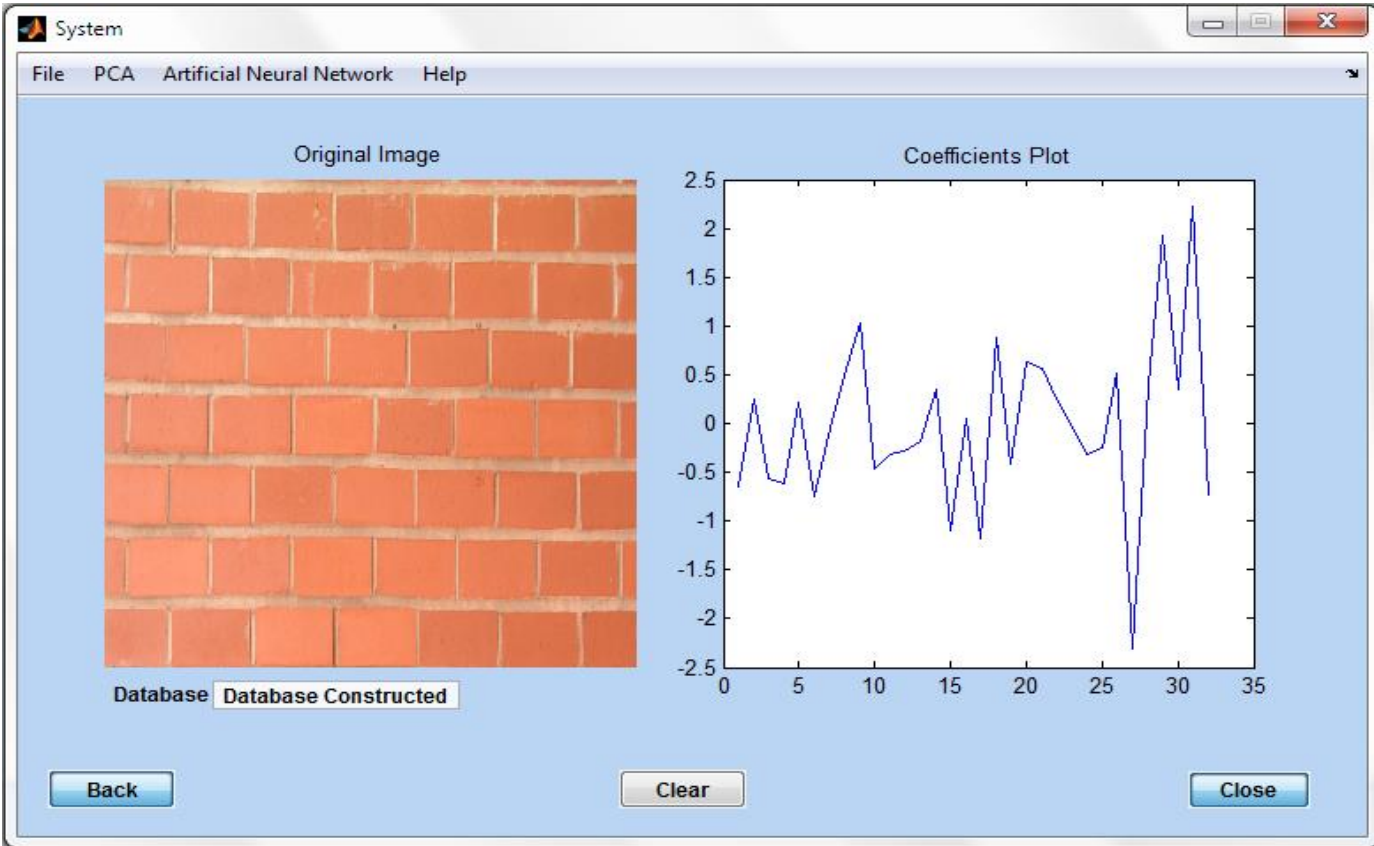

الثكل (11): معاملات تحليل المكونات الأساسية

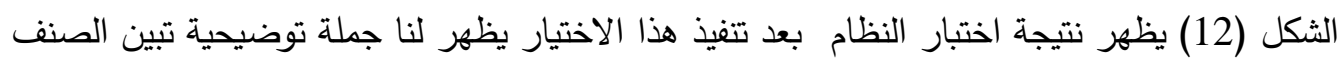

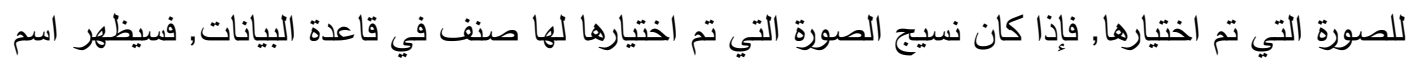
الصنف الذي ينتمي إليه نسيج هذه الصورة. 


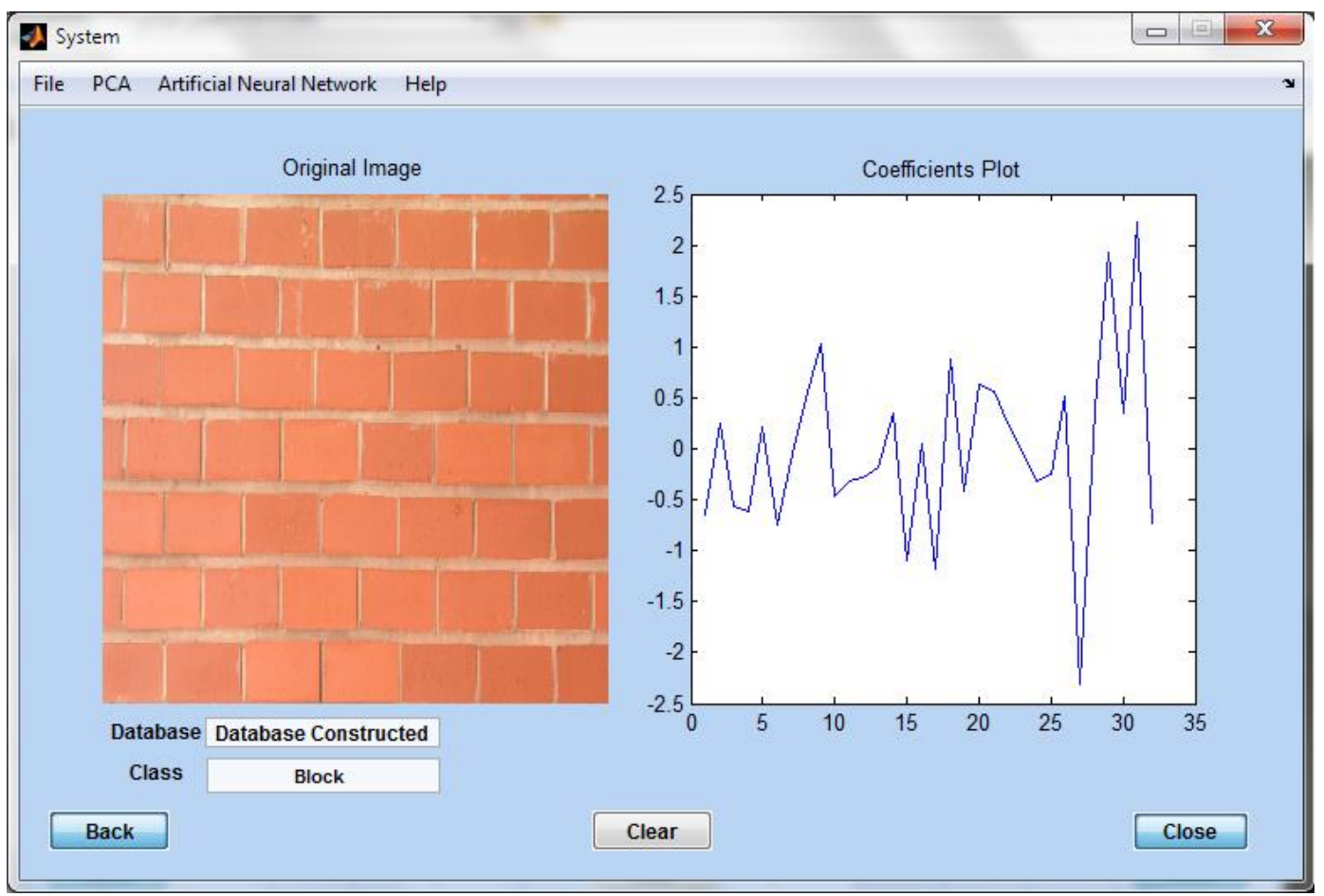

الثكل (12): تنفيذ التمييز

بينما إذا تم اختيار نسيج صورة غير موجود في قاعدة البيانات, فسيظهر جملة تؤكد عدم مقدرة النظام

$$
\text { على تمييز هذا النسيج كما موضح في الثكل التالي: }
$$

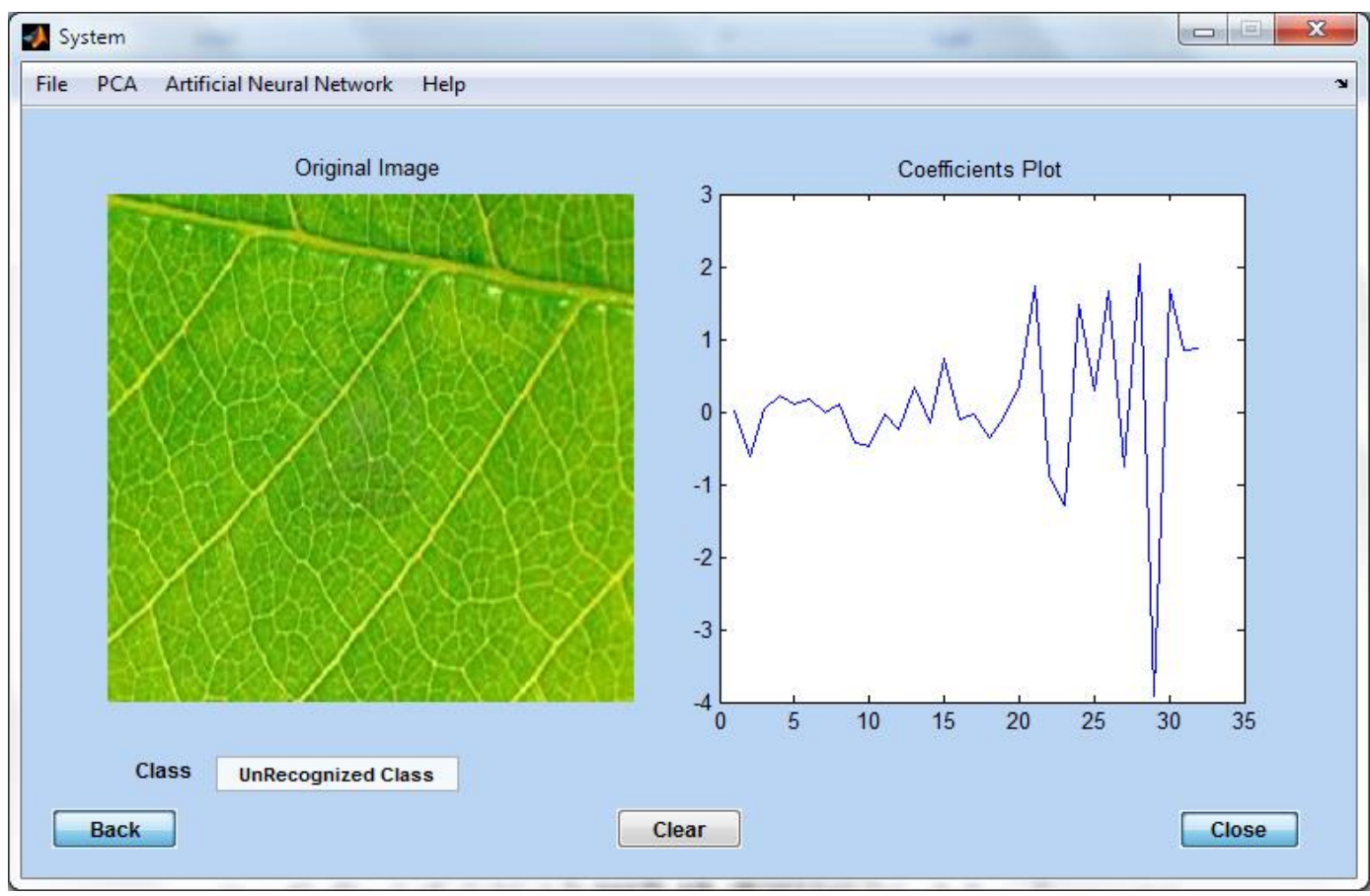

الثكل (13): نسيج لا يمكن تمييزه 


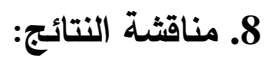

بعد تتفيذ مرحلة الاختبار على النظام تم الحصول على نتائج دونت في الجدول (1) حيث تم اختبار 94

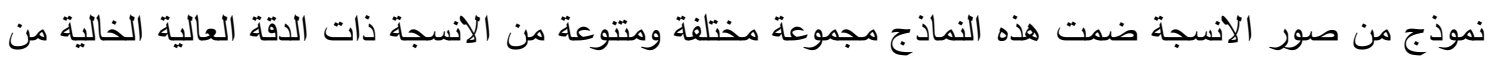

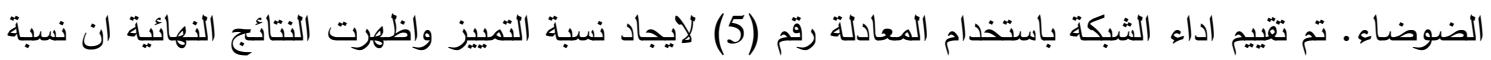
التمييز ممتازة حيث بلغت 92\% وان الاختلاف في نسب التمييز بين انواع الاصناف يعتمد على مخرجات خوارزمية تحليل المركبات الاساسية والتي تتاثر باتجاه النسجة في الصورة. الجدول (1) نتائج تنفيذ النظام

\begin{tabular}{|c|c|c|c|}
\hline نسبة التمييز (RR) & التمييز الخاطئ & التمييز الصحيح & اسم الصنف \\
\hline$\% 80$ & 2 & 8 & نسيج الخشب المسطح \\
\hline$\% 90$ & 1 & 9 & نسيج الحجر \\
\hline$\% 80$ & 2 & 8 & نسيج الطابوق \\
\hline$\% 100$ & 0 & 10 & نسيج الفاكهة \\
\hline$\% 90$ & 1 & 9 & نسيج نجارة الخشب \\
\hline$\% 100$ & 0 & 8 & نسيج أرضية منزل \\
\hline$\% 100$ & 0 & 8 & نسيج ورق حائط \\
\hline$\% 100$ & 0 & 10 & نسيج إسفلت \\
\hline$\% 90$ & 1 & 9 & نسيج خشب بشكل ألواح \\
\hline$\% 100$ & 0 & 8 & نسيج الرخام \\
\hline$\% 92$ & 7 & 87 & المجموع \\
\hline
\end{tabular}

9. 9. الاسـتنتاجات

1- ان استخدام صور الانسجة ذات الدقة العالية الخالية من الضوضاء ساعد على الحصول على الدقة في التمييز 2- ان استخدام خوارزمية تحليل المركبات الاساسية ادى الى تقليل حجم البيانات الداخلة للشبكة العصبية

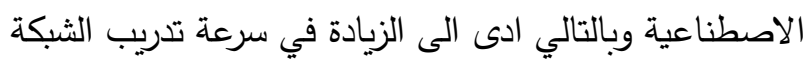
3- اثبتت الشبكة العصبية ELMAN كفاءتها العالية في عملية التمييز 4- ان استخدام خوارزمية تحليل المركبات الاساسية اضاف كفاءة ودقة في تمييز الانسجة بالرغم من التشابه النسبي الموجود بين الانسجة 


\section{المصادر}

[1] S. Aggarwl, A. Jangra, (2011), "Image Quality Assessment Based on Feature Extraction Using Wavelet and Riesz Transforms" International Journal of Computer Science and Security (IJCSS), Volume (1).

[2] Imran S. Bajwa, S. Irfan Hyder, (2005), " PCA Based Classification of Single-layered Cloud Types", Vol 1 (N.2).

[3] Y.H. Zhang, C.W.M. Yuen, W.K. Wong, (2010),"A New Intelligent Fabric Defect Detection And Classification system Based on Gabor Filter and Modified Elman Neural Network", IEEE, pp. 652-656.

[4] Othman, M. F., Basri, M. A. M., (2011),"Probabilistic Neural Network for Brain Tumor Classification", Second International Conference on Intelligent Systems, Modelling and Simulation, pp. 136-138.

[5] Ghassem F., 21 February, (2012)," A New Intelligent Fabric Defect Detection And Classification system Based on Gabor Filter and Modified Elman Neural Network", African Journal of Business Management, Vol. 6 (33), pp. 9496-9503.

[6] Turkan A. Khalel,(2001),"An Integrated Software for Remotly Sensed Data Classification", Thesis, University of Mosul-Iraq.

[7] Jamal S., Sora R. Sharef, Osama Y. Mohamad ,(2011)," An Intelligent Computer System Recognizing Printed Russian Letters Using Artificial Neural Network", IASJ ,Vol(8), Pgs.125-144.

[8] Maha A. Alhaso, Muna Jafar , (2010)," Choosing best band by using PCA algorithm on remote sensing ", IASJ, vol. 7, issue: 3,p.p 135-144

[9] Jamal S., Osama Y. Mohamad, Tariq H., (2010),"Recognizing Hebrew Letters Using Artificial Neural Network", Bhoth Mostakbaliya Journal, Pgs. 143-175.

[10] Li. Penghua Chai,(2013),'Quantized Neural Modeling:Hybrid Quantized Architecture in Elman Networks",Vol.37,ISSUE2,Neural Computing \& application.

[11] Howard Demuth, Mark Beale, R2007b, "Neural Network Toolbox".

[12] Fawcett Tom. (2004),"Roc graphs: Notes and practical considerations for researchers". Technical Report, HP Laboratories, Kluwer Academic Publishers. 